\title{
Brain Shift Computation Using a Fully Nonlinear Biomechanical Model
}

\author{
Adam Wittek $^{1}$, Ron Kikinis ${ }^{2}$, Simon K. Warfield ${ }^{3}$, and Karol Miller ${ }^{1}$ \\ ${ }^{1}$ Intelligent Systems for Medicine Laboratory, \\ School of Mechanical Engineering, The University of Western Australia, \\ 35 Stirling Highway, Crawley/Perth WA 6009 Australia \\ 2 Surgical Planning Laboratory, \\ ${ }^{3}$ Computational Radiology Laboratory, \\ Brigham and Women's Hospital, Children's Hospital and Harvard Medical School, \\ 75 Francis Street, Boston, MA02115, USA \\ Karol Miller: kmiller@mech.uwa.edu.au \\ http: / /www. mech. uwa.edu.au/ISML
}

\begin{abstract}
In the present study, fully nonlinear (i.e. accounting for both geometric and material nonlinearities) patient specific finite element brain model was applied to predict deformation field within the brain during the craniotomyinduced brain shift. Deformation of brain surface was used as displacement boundary conditions. Application of the computed deformation field to align (i.e. register) the preoperative images with the intraoperative ones indicated that the model very accurately predicts the displacements of gravity centers of the lateral ventricles and tumor even for very limited information about the brain surface deformation. These results are sufficient to suggest that nonlinear biomechanical models can be regarded as one possible way of complementing medical image processing techniques when conducting nonrigid registration. Important advantage of such models over the linear ones is that they do not require unrealistic assumptions that brain deformations are infinitesimally small and brain tissue stress-strain relationship is linear.
\end{abstract}

\section{Introduction}

Brain shift occurring during craniotomy distorts the preoperative anatomy and often leads to misalignment between the actual position of important brain structures and its position determined from the preoperative images. This is one of the key challenges faced by neurosurgery, and its importance is increasing with progress of therapeutic technologies. Important feature of the technologies that are entering medical practice now, such as e.g. gene therapy, nanotechnology devices, focused radiation, lesion generation and robotic surgery, is that they have extremely localized area of therapeutic effect [1]. Therefore, they have to be applied precisely in relation to the current (i.e. intraoperative) patient's anatomy, directly over specific location of anatomic/functional abnormality (e.g. tumor).

While the intraoperative imaging would be the most straightforward method when determining the current position of the tumor during surgery, its quality suffers from the constraints of the operating room. As a result of these constraints, spatial resolu- 
tion and contrast of intraoperative images are typically inferior to those of preoperative ones [2]. This problem is typically solved by aligning (i.e., registering) the preoperative data to scans of the brain acquired intraoperatively, which makes it possible to retain the preoperative image quality during the surgery. In order to achieve accurate alignment, the brain deformation must be taken into account, which implies nonrigid registration. In recent years, biomechanical models have been recognized and used as efficient tools for predicting the brain deformation. In most practical cases, such models utilize the finite element method [3] to solve sets of partial differential equations governing the deformation behavior of continua.

Selection of appropriate mathematical model governing the deformation is crucial to ensure realistic computer simulation of brain deformation. In nonrigid registration, linear elastic formulation is typically used [2], [4], [5]. In this formulation, the brain deformations are regarded as infinitesimal (i.e. geometric linearity) and brain tissue is treated as an elastic material in which the stress is a linear function of the strain (i.e. material linearity). Although nonrigid registration using linear biomechanical models has been an important achievement that significantly adds to the value of intraoperative imaging [2], it must be realized that neither the assumption about infinitesimally small brain deformation nor the one about brain stress-strain linear behavior is valid during brain shift. Craniotomy typically results in deformation of brain surface as large as $10 \mathrm{~mm}$ [6] (i.e. around $10 \%$ of distance between the left and right cortical landmarks) and rigid body movement of the brain, which implies that fully nonlinear finite element formulations and material models are needed when predicting deformation field within the brain during a typical brain shift.

Therefore, we propose that when conducting nonrigid registration, image analysis techniques should be complemented by biomechanical models based on fully nonlinear finite element formulations rather than linear ones. We demonstrate that such nonlinear models facilitate accurate prediction of deformation field within the brain even when simplified brain geometry and limited data about the brain surface deformation are used.

\section{Methods}

\subsection{Construction of Finite Element Mesh for Patient Specific Brain Model}

In the present study, a volumetric (i.e. three-dimensional) patient specific brain mesh was constructed from the segmented preoperative magnetic resonance images (MRIs) (Fig. 1). The segmentation was done using 3D Slicer [7] — open-source software for visualization, registration, segmentation and quantification of medical data developed by Artificial Intelligence Laboratory of Massachusetts Institute of Technology and Surgical Planning Laboratory at Brigham and Women's Hospital and Harvard Medical School.

The mesh was built using 15036 hexahedron elements (i.e. 8-node "bricks") (Fig. 2). The hexahedron finite elements are known to be the most effective ones in nonlinear finite element procedures using explicit time integration. The construction of hexahedron grid for the present brain model has been described in our previous publication [8]. 
In order to simulate the pia matter, the brain surface was covered by a layer of 3522 membrane elements (thickness of $0.1 \mathrm{~mm}$ ). The cerebral falx was also discretized using membrane elements: 436 such elements of thickness of $1 \mathrm{~mm}$ were used.

a)

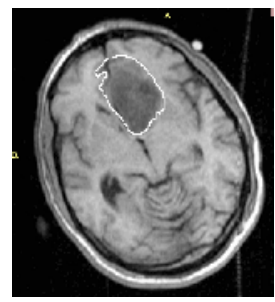

b)

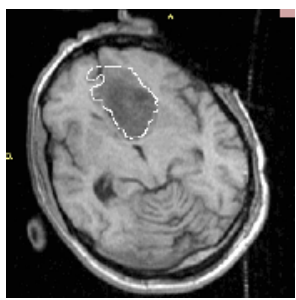

Fig. 1. Example of segmented MRIs of the head used when building patient specific brain mesh. The tumor segmentation is indicated by white lines in the anterior brain part. a) Preoperative; b) Intraoperative.

a)

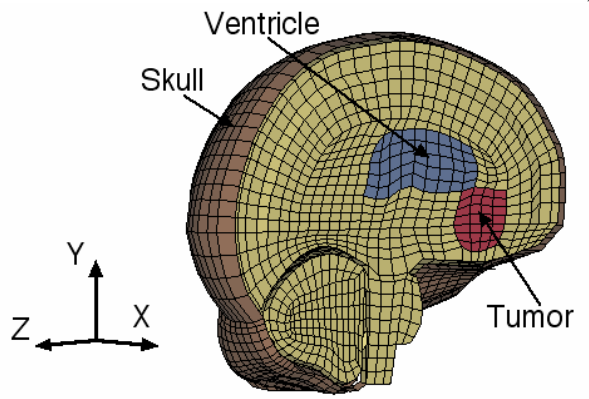

b)

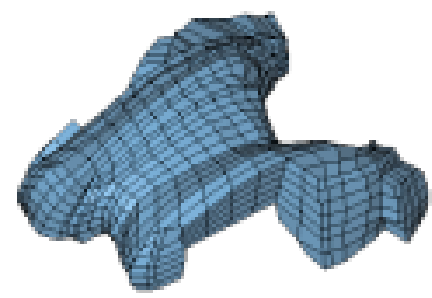

Fig. 2. Patient specific brain mesh constructed in the present study. a) Entire left brain hemisphere; b) Lateral ventricles.

\subsection{Brain Shift Simulation}

Equations of Mathematical Model. Detailed account of biomechanics of the brain including relevant equations was given by Miller [9]. In this section, the basic ideas are summarized.

From the perspective of surgical simulation, the brain can be considered a singlephase continuum undergoing large deformations. In the present analysis, the stresses and strains were measured with respect to the current configuration. Thus, energetically conjugate Almansi strain $\boldsymbol{e}$ and Cauchy stress $\boldsymbol{\tau}$ (i.e. forces per unit areas in the deformed geometry) were used:

$$
\boldsymbol{e}=\frac{1}{2}\left[\boldsymbol{I}-\left(\boldsymbol{F}^{-1}\right)^{T}\left(\boldsymbol{F}^{-1}\right)\right],
$$

where $\boldsymbol{F}$ is the deformation gradient and $\boldsymbol{I}$ is the identity matrix. 
Using Almansi strain and Cauchy stress, the equation of equilibrium can be written in the following way:

$$
\tau_{, i}^{i j}+\rho R_{i}=0
$$

where $\rho$ is a mass density, $R_{i}$ is a body force per unit mass in direction $i$, and comma indicates covariant differentiation with respect to the deformed configuration. Repeated index summation convention was used.

Eqs. (1)-(2) must be supplemented by formulae describing mechanical properties of materials, i.e. appropriate constitutive models. Modeling of physical properties of the brain is still an uncovered area pioneered by a few only [10], [11], [12]. As shown by Miller and Chinzei [13], [14], the stress-strain behavior of the brain tissue is nonlinear. The stiffness in compression is significantly higher than in extension. One can also observe a strong stress-strain rate dependency. To account for these complexities, we used the model suggested by Miller and Chinzei [14]:

$$
\begin{gathered}
W=\frac{2}{\alpha^{2}} \int_{0}^{t}\left[\mu(t-\tau) \frac{\mathrm{d}}{\mathrm{d} \tau}\left(\lambda_{1}^{\alpha}+\lambda_{2}^{\alpha}+\lambda_{3}^{\alpha}-3\right)\right] \mathrm{d} \tau, \\
\mu=\mu_{0}\left[1-\sum_{k=1}^{n} g_{k}\left(1-e^{-\frac{t}{\tau_{k}}}\right)\right],
\end{gathered}
$$

where $W$ is a potential function, $\lambda_{\mathrm{i}}$ 's are principal stretches, $\mu_{0}$ is the instantaneous shear modulus in undeformed state, $\tau_{k}$ are characteristic times, $g_{k}$ are relaxation coefficients, and $\alpha$ is a material coefficient, which can assume any real value without restrictions. The model parameters are given in Table 1 .

The cerebral falx was assigned Young's modulus of $3.4 \mathrm{MPa}$ [15]. For pia, the Young's modulus of 1.1 MPa was used - a value derived from the studies on brain injury by Takhounts et al. [15] and Zhang et al. [16].

Table 1. List of material constants for constitutive model of brain tissue, Eqs. (3) and (4), $n=2$. The constants were taken from Miller and Chinzei [14]

\begin{tabular}{c|l}
\hline Instantaneous response & $\mu_{0}=842[\mathrm{~Pa}] ; \alpha=-4.7$ \\
\hline$k=1$ & Characteristic time $\tau_{l}=0.5[\mathrm{~s}] ; g_{l}=0.450$ \\
\hline$k=2$ & Characteristic time $\tau_{2}=50[\mathrm{~s}] ; g_{2}=0.365$ \\
\hline
\end{tabular}

Integration of Equations of Equilibrium. Integration of equations of equilibrium/dynamics (i.e. Eq. 2) can be done using either implicit or explicit methods [3]. The implicit integration methods are unconditionally stable but can be time consuming as iterations are conducted at each time step. Therefore, in the present study an explicit integration was used. In the explicit integration, no iteration is needed as the displacement at time $t+\Delta t$ is solely based on the equilibrium at time $t$.

The computations were conducted using the LS-DYNA code (Livermore Software Technology Corporation, Livermore, California, USA) [17], which is one of the explicit finite element codes routinely applied in car crash simulation. Fully nonlinear 
formulations are used in $L S-D Y N A$, i.e. both geometrical and material nonlinearities are taken into account.

Boundary Conditions for Simulation of Brain Shift. The anterior parts of the preoperative and intraoperative cortical surfaces were discretized using consistent rectangular meshes of the same density. The distances between corresponding nodes of the preoperative and intraoperative cortical surfaces were calculated and used as displacement boundary conditions (i.e. prescribed nodal displacements) for the nodes located in the anterior part of the brain model surface. To define the boundary conditions for the remaining nodes on the brain model surface, contact interfaces were defined between the rigid skull model and parts of the brain surface where the nodal displacements were not prescribed.

The spine-spinal cord interactions and constraining effects of the spinal cord on the brain rigid body motion were simulated by rigidly constraining the spinal end of the model.

In biomechanical models for nonrigid registration, cerebral falx is typically not simulated. However, as suggested by Warfield [18], disregarding falx may lead to misregistration of the lateral ventricles on the side opposite to surgical intervention. Therefore, in the present study the falx was simulated as elastic membrane rigidly attached to the skull. The contact interfaces were defined between this membrane and inter-hemisphere surfaces.

\section{Results}

The craniotomy-induced displacements of the ventricles' and tumor's centers of gravity (COGs) predicted by the model agreed well with the actual ones determined from the radiographic images (Table 2). With the exception of the tumor COG displacement along the $\mathrm{Y}$ (i.e. inferior-superior) axis, the differences between the computed and observed displacements were below $0.65 \mathrm{~mm}$. Important and not unexpected feature of the results summarized in Table 2 is that the displacements of the tumor's and ventricles' COGs appreciably differed. This feature can be explained only by the fact that the brain undergoes both local deformation and global rigid body motion, which implies that nonrigid registration had to be used.

Table 2. Comparison of craniotomy-induced displacements of ventricles' and tumor's centers of gravity (COGs) predicted by the present brain model with the actual ones determined from MRIs. Directions of $\mathrm{X}, \mathrm{Y}$ and $\mathrm{Z}$ axes are given in Fig. 2a.

\begin{tabular}{l|c|c}
\hline & Determined from MRIs & Predicted \\
\hline \multirow{3}{*}{ Ventricles } & $\Delta \mathrm{x}=3.40 \mathrm{~mm}$ & $\Delta \mathrm{x}=3.06 \mathrm{~mm}$ \\
& $\Delta \mathrm{y}=0.25 \mathrm{~mm}$ & $\Delta \mathrm{y}=0.29 \mathrm{~mm}$ \\
& $\Delta \mathrm{z}=1.73 \mathrm{~mm}$ & $\Delta \mathrm{z}=1.65 \mathrm{~mm}$ \\
\hline \multirow{3}{*}{ Tumor } & $\Delta \mathrm{x}=5.36 \mathrm{~mm}$ & $\Delta \mathrm{x}=4.74 \mathrm{~mm}$ \\
& $\Delta \mathrm{y}=-3.52 \mathrm{~mm}$ & $\Delta \mathrm{y}=-0.40 \mathrm{~mm}$ \\
& $\Delta \mathrm{z}=2.64 \mathrm{~mm}$ & $\Delta \mathrm{z}=2.77 \mathrm{~mm}$ \\
\hline
\end{tabular}




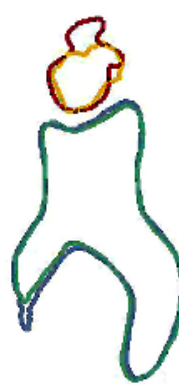

$-12.5 \mathrm{~mm}$
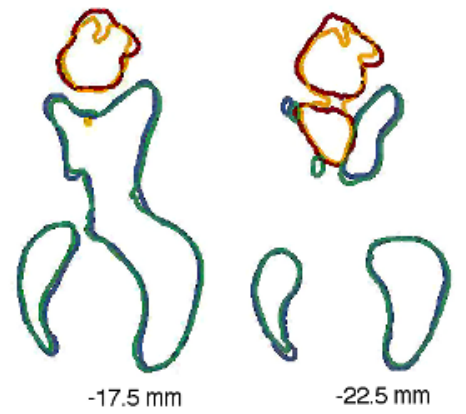

Explanation:

- Tumor simulation

-Ventricle simulation

Tumor intraoperative

image

- Ventricle intraoperative

Fig. 4. Comparison of contours of axial sections of ventricles and tumor obtained from the intraoperative images with the ones predicted using the present brain model. Positions of section cuts are measured from the most superior point of parietal cortex (superior direction is positive).

Detailed comparison of cross sections of the actual tumor and ventricle surfaces acquired intraoperatively with the ones predicted by the present brain model indicates some local misregistration, particularly in the inferior tumor part (Fig. 4). However, the overall agreement is remarkably good.

\section{Discussion and Conclusions}

Instead of relying on unrealistic linearization (i.e. assumption about infinitesimally small brain deformation during craniotomy and linear stress - strain relationship of brain tissue) used in almost all biomechanical models for neurosurgical registration (with a notable exception of $\mathrm{Xu}$ and Nowinski [19]), we applied fully nonlinear (i.e. including both geometrical and material nonlinearities) finite element formulations to compute deformation field within the brain. These formulations made it possible to predict the displacement of the ventricles' and tumor's centers of gravity with around $0.5 \mathrm{~mm}$ accuracy (Table 2). This remarkable accuracy was achieved using displacement boundary conditions determined from very limited information about the brain surface deformation.

Detailed comparison of the calculated and image-determined cross sections of ventricles and tumor after craniotomy indicated local misregistration (Fig. 4), but the overall agreement was remarkably good. The local inaccuracies observed here could be related to simplifications of the brain geometry when building the finite element mesh and inability of our brain model to accurately account for various complex physiological phenomena, such as loss of fluid from ventricles, that could affect deformation field within the brain. A combined approach is needed in which nonlinear biomechanical models, such as the one developed in the present study, are applied together with traditional registration methods relying on image processing techniques, such as e.g. optical flow [20], mutual information-based similarity [21], entropybased alignment [22] and block matching [23].

The presented results indicate that finite element analysis using fully nonlinear solid mechanics formulations is a powerful method for computing deformation field within the brain during craniotomy. As realistic prediction of deformation field within 
the brain is crucial for nonrigid registration, our results show that biomechanical models using fully nonlinear finite element formulations can be regarded as promising tool when complementing traditional image processing techniques used in image registration. Such models do not require unrealistic assumptions that the brain deformations are infinitesimally small and brain tissue stress-strain relationship is linear. Therefore, they can be seen as one possible way of improving reliability of image registration.

\section{Acknowledgements}

The financial support of the Australian Research Council (Grant No. DP0343112) is gratefully acknowledged.

The medical images used in the present study were obtained in the investigation supported by NSF ITR 0426558, a research grant from the Whitaker Foundation and by NIH grants R21 MH67054, R01 LM007861, P41 RR13218 and P01 CA67165.

The authors gratefully acknowledge collaboration of Toyota Central R\&D Labs. (Nagakute, Aichi, Japan) in development of brain model.

\section{References}

1. Bucholz, R., MacNeil, W. and McDurmont, L.: The operating room of the future. Clinical Neurosurgery, Vol. 51 (2004) 228-237

2. Warfield, S. K., Talos, F., Tei, A., Bharatha, A., Nabavi, A., Ferrant, M., Black, P. McL., Jolesz, F. A. and Kikinis, R.: Real-time registration of volumetric brain MRI by biomechanical simulation of deformation during image guided surgery. Computing and Visualization in Science, Vol. 5 (2002) 3-11

3. Bathe, K.-J.: Finite Element Procedures. Prentice-Hall (1996)

4. Ferrant, M., Nabavi, A., Macq, B., Black, P. McL., Jolesz, F. A., Kikinis, R. and Warfield, S. K.: Serial registration of interoperative MR images of the brain. Medical Image Analysis, Vol. 6 (2002) 337-359

5. Hagemann, A., Rohr, K., Stiehl, H. S., Spetzger, U. and Gilsbach, J. M.: Biomechanical modeling of the human head for physically based, nonrigid image registration. IEEE Transactions on Medical Imaging, Vol. 18 (1999) 875-884

6. Miga, M. I., Sinha, T. K., Cash, D. M., Galloway, R. L. and Weil, R. J.: Cortical surface registration for image-guided neurosurgery using laser-range scanning. IEEE Transactions on Medical Imaging, Vol. 22 (2003) 973-985

7. 3D Slicer: Medical visualization and processing environment for research, http://www.slicer.org/

8. Wittek, A., Miller, K., Laporte, J., Kikinis, R. and Warfield, S. K.: Computing reaction forces on surgical tools for robotic neurosurgery and surgical simulation, in Proc. of Australasian Conference on Robotics and Automation ACRA, Canberra, Australia, (2004) 1-8

9. Miller, K.: Biomechanics of Brain for Computer Integrated Surgery. Warsaw: Publishing House of Warsaw University of Technology (2002)

10. Miller, K. and Chinzei, K.: Modeling of soft tissues deformation. Journal of Computer Aided Surgery, Vol. 1 (1995) 62-63

11. Bilston, L. E., Liu, Z. and Phan-Tien, N.: Linear viscoelastic properties of bovine brain tissue in shear. Biorheology, Vol. 34 (1997) 377-385 
12. Prange, M. T. and Margulies, S. S.: Regional, directional, and age-dependent properties of the brain undergoing large deformation. ASME Journal of Biomechanical Engineering, Vol. 124 (2002) 244-252

13. Miller, K. and Chinzei, K.: Constitutive modelling of brain tissue; Experiment and Theory. Journal of Biomechanics, Vol. 30 (1997) 1115-1121

14. Miller, K. and Chinzei, K.: Mechanical properties of brain tissue in tension. Journal of Biomechanics, Vol. 35 (2002) 483-490

15. Takhounts, E. G., Eppinger, R. H., Campbell, J. Q., Tannous, R. E., Power, E. D. and Shook, S. S.: On the development of the SIMon finite element head model. Stapp Car Crash Journal, Vol. 47 (2003) 107-133

16. Zhang, L., Bae, J., Hardy, W. N., Monson, K. L., Manley, G. T., Goldsmith, W., Yang, K. H. and King, A. I.: Computational study of the contribution of the brain vasculature on the dynamic response of the brain. Stapp Car Crash Journal, Vol. 46 (2002) 145-163

17. Hallquist, J. O.: LS-DYNA Theoretical Manual, Livermore Software Technology Corporation (1998)

18. Warfield, S. K., Ferrant, M., Gallez, X., Nabavi, A., Jolesz, F. A. and Kikinis, R.: Real-time biomechanical simulation of volumetric brain deformation for image guided neurosurgery, in Proc. of SC 2000: High Performance Networking and Computing Conference, Dallas, USA, (2000) 1-16

19. Xu, M. and Nowinski, W. L.: Talairach-Tournoux brain atlas registration using a metalforming principle-based finite element method. Medical Image Analysis, Vol. 5 (2001) 271-279

20. Beauchemin, S. S. and Barron, J. L.: The computation of optical flow. ACM Computing Surveys, Vol. 27 (1995) 433-467

21. Viola, P. A.: Alignment by maximization of mutual information. Artificial Intelligence Laboratory, Massachusetts Institute of Technology, Report AI-TR-1548 (1995)

22. Warfield, S. K., Rexilius, J., Huppi, P. S., Inder, T. E., Miller, E. G., Wells III, W. M., Zientara, G. P., Jolesz, F. A. and Kikinis, R.: A binary entropy measure to assess nonrigid registration algorithms, in Proc. of MICCAI 2001: Fourth International Conference on Medical Image Computing and Computer Assisted Intervention, Utrecht, The Netherlands, (2001) 266-274

23. Dengler, J. and Schmidt, M.: The dynamic pyramid - a model for motion analysis with controlled continuity. International Journal of Pattern Recognition and Artificial Intelligence, Vol. 2 (1988) 275-286 